\title{
Some properties of edge intersection graphs of single-bend paths on a grid
}

\author{
A. Asinowski ${ }^{\mathrm{a}, \mathrm{b}}, \mathrm{B}$. Ries ${ }^{\mathrm{c}, *}$ \\ a Caesarea Rothschild Institute, University of Haifa, Haifa 31905, Israel \\ ${ }^{\mathrm{b}}$ Department of Mathematics, Technion-Israel Institute of Technology, Israel \\ ${ }^{\mathrm{c}}$ LAMSADE, Department of Mathematics and Computer Science, Université Paris-Dauphine, Place du Maréchal de Lattre de Tassigny, \\ 75775 PARIS Cedex 16, France
}

\section{A R T I C L E I N F O}

\section{Article history:}

Received 14 September 2010

Received in revised form 4 October 2011

Accepted 5 October 2011

Available online 27 October 2011

\section{Keywords:}

Intersection graphs

Paths on a grid

Chordal graphs

Neighborhood

Erdős-Hajnal property

\begin{abstract}
A B S T R A C T
In this paper we consider graphs $G$ whose vertices can be represented as single-bend paths (i.e., paths with at most one turn) on a rectangular grid, such that two vertices are adjacent in $G$ if and only if the corresponding paths share at least one edge of the grid. These graphs, called $B_{1}$-EPG graphs, were first introduced in Golumbic et al. (2009) [13]. Here we show that the neighborhood of every vertex in a $B_{1}$-EPG graph induces a weakly chordal graph. From this we conclude that the family $\mathcal{F}$ of $B_{1}$-EPG graphs satisfies the Erdős-Hajnal property with $\epsilon(\mathcal{F})=\frac{1}{3}$, i.e., that every $B_{1}$-EPG graph on $n$ vertices contains either a clique or a stable set of size at least $n^{\frac{1}{3}}$. Finally we give a characterization of $B_{1}$-EPG graphs among some subclasses of chordal graphs, namely chordal bull-free graphs, chordal clawfree graphs, chordal diamond-free graphs, and special cases of split graphs.
\end{abstract}

(C) 2011 Elsevier B.V. All rights reserved.

\section{Introduction}

Edge intersection graphs of paths on a grid (or for short EPG graphs) are graphs whose vertices can be represented as paths on a rectangular grid such that two vertices are adjacent if and only if the corresponding paths share at least one edge of the grid. These graphs were first introduced in [13] and have also been studied by several authors (see $[2,6,14]$ ). The motivation for studying these graphs comes from circuit layout problems (see for instance [3]). In [13] the authors show that every graph $G$ is an EPG graph. They also introduce some subclasses of EPG graphs, namely $B_{k}$-EPG graphs, for $k \geq 0$. For these graphs, the paths on the grid that represent the vertices of $G$ are allowed to have at most $k$ bends, i.e., at most $k$ turns. They show that every tree is a $B_{1}$-EPG graph and they also give some examples of graphs which are not $B_{1}$-EPG. Furthermore, the representation of cliques and cycles in $B_{1}$-EPG graphs is considered. In [2] the authors study questions related to the size of the grid that is needed in order to represent every $n$ vertex graph as an edge intersection of paths on a grid. Furthermore, they show that for any $k$, only a small fraction of all labeled graphs on $n$ vertices are $B_{k}$-EPG. Some results of [2] were also proved in [6]. In addition the authors in [6] consider different classes of graphs and show in particular that every planar graph is a $B_{5}$-EPG graph. In [14], the authors prove that recognizing $B_{1}$-EPG graphs is $\mathcal{N} \mathcal{P}$-complete.

In this paper we focus on $B_{1}$-EPG graphs. The paper is organized as follows. In Section 2 we give definitions and notation as well as some useful results from [13]. In Section 3 we present some properties of the neighborhood of a vertex in a $B_{1}$-EPG graph. In Section 4 we prove that $B_{1}$-EPG graphs satisfy the Erdős-Hajnal property with $\epsilon(\mathcal{F})=\frac{1}{3}$, i.e., that every $B_{1}$-EPG graph on $n$ vertices contains either a clique or a stable set of size at least $n^{\frac{1}{3}}$. Section 5 focuses on some subclasses of chordal

\footnotetext{
* Corresponding author.

E-mail addresses: andrei@tx.technion.ac.il (A. Asinowski), bernard.ries@dauphine.fr (B. Ries).
} 

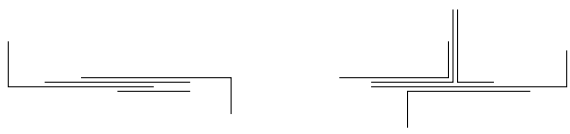

Fig. 1. Left: an edge clique. Right: a claw clique.

graphs and gives a characterization of $B_{1}$-EPG graphs of these subclasses. Finally we conclude with some open questions in Section 6. For graph theoretical terms that are not defined here, we refer the reader to [4].

\section{Preliminaries}

All graphs in this paper are finite and simple. Let $G=(V(G), E(G))$ be a graph. For a vertex $v \in V(G)$, we let $N_{G}(v)$ denote the set of vertices in $G$ that are adjacent to $v$, i.e., the neighbors of $v . N_{G}(v)$ is called the neighborhood of vertex $v$. We will write $N_{G}[v]=N_{G}(v) \cup\{v\}$, and call $N_{G}[v]$ the closed neighborhood of vertex $v$. Whenever it is clear from the context what $G$ is, we will drop the subscripts and write $N(v)=N_{G}(v)$ and $N[v]=N_{G}[v]$. A clique is a set of pairwise adjacent vertices and a stable set is a set of pairwise nonadjacent vertices. For $X \subseteq V(G)$, we denote by $G \mid X$ the subgraph induced by $X$. For graphs $G$ and $H, G$ is said to be $H$-free if $G$ has no induced subgraph isomorphic to $H$.

For disjoint sets $A, B \subseteq V(G)$, we say that $A$ is complete to $B$ if every vertex in $A$ is adjacent to every vertex in $B$, and that $A$ is anticomplete to $B$ if every vertex in $A$ is nonadjacent to every vertex in $B$. A hole is an induced cycle on at least four vertices. An antihole is the complement of a hole. The length of a hole (antihole) is the number of vertices inducing the hole (antihole). A hole (antihole) is odd if it has odd length. A graph $G$ is chordal if $G$ does not contain any hole. A graph $G$ is weakly chordal if it contains no hole of length at least 5 and no antihole of length at least 5 . For a graph $G$, let $\omega(G)$ denote the size of a largest clique in $G$, and let $\chi(G)$ denote the chromatic number of $G$. A graph $G$ is perfect if $\chi\left(G^{\prime}\right)=\omega\left(G^{\prime}\right)$ for every induced subgraph $G^{\prime}$ of $G$. It has been shown in [7] that a graph is perfect if and only if it contains no odd hole and no odd antihole. Three vertices $u, v, w$ of a graph $G$ form an asteroidal triple (AT) of $G$ if for every pair of them there exists a path connecting the two vertices and such that the path avoids the neighborhood of the remaining vertex. We denote by $P_{k}$ an induced path on $k$ vertices, and by $H_{k}$ a hole on $k$ vertices. The complement of a graph $G$ is denoted by $\bar{G}$.

Let $g$ be a rectangular grid of size $(2 m+1) \times(2 m+1)$. The horizontal grid lines will be referred to as rows and denoted by $y_{-m}, y_{-m+1}, \ldots, y_{0}, \ldots, y_{m-1}, y_{m}$, and the vertical grid lines will be referred to as columns and denoted by $x_{-m}, x_{-m+1}, \ldots, x_{0}, \ldots, x_{m-1}, x_{m}$. Let $\mathcal{P}$ be a collection of nontrivial simple paths on $g$. We define the edge intersection graph $\operatorname{EPG}(\mathcal{P})$ of $\mathcal{P}$ to have vertices which correspond to the members of $\mathcal{P}$, such that two vertices are adjacent in $\operatorname{EPG}(\mathcal{P})$ if and only if the corresponding paths in $\mathcal{P}$ share at least one edge in $g$. An undirected graph $G$ is called an edge intersection graph of paths on a grid $(E P G)$ if $G=\operatorname{EPG}(\mathcal{P})$ for some $\mathcal{P}$ and $\mathcal{G}$, and $\langle\mathcal{P}, \mathcal{g}\rangle$ is an EPG representation of $G$. For any vertex $v \in V(G)$, we denote by $P_{v}$ the corresponding path in the EPG representation of $G$. In this paper we will always assume that the size of the grid $g$, in particular $m$, is sufficiently large such that the $B_{1}$-EPG graphs that we are interested in admit an EPG representation on $g$.

A turn of a path at a grid point is called a bend and the grid point is called a bend point. An EPG representation is a $B_{k}$-EPG representation if each path has at most $k$ bends. A graph that has a $B_{k}$-EPG representation is called $B_{k}$-EPG. In this paper we only consider $B_{1}$-EPG graphs. We define a $\left\ulcorner\right.$-path $P$ as a bended path with some bend point $\left(x_{i}, y_{j}\right)$ such that $P$ uses column $x_{i}$ between rows $y_{k}$ and $y_{j}$, for some $k<j$, and $P$ uses row $y_{j}$ between columns $x_{i}$ and $x_{l}$, for some $l>i$. ᄀ-paths, ᄂ-paths, and \lrcorner -paths are defined in a similar way. We say that a path $P$ on the grid contains a grid point $\left(x_{i}, y_{j}\right)$ if $\left(x_{i}, y_{j}\right) \subseteq P$ and $\left(x_{i}, y_{j}\right)$ is not an endpoint of $P$. A horizontal segment (resp. vertical segment) on a row $y$ (resp. a column $x$ ) between columns $x_{i}$ and $x_{j}, i<j$ (resp. between rows $y_{p}$ and $\left.y_{q}, p<q\right)$ is denoted by $\left[x_{i}, x_{j}\right] \times\{y\}$ (resp. $\left.\left[y_{p}, y_{q}\right] \times\{x\}\right)$.

Let $K$ be a clique of a $B_{1}$-EPG graph $G$. If there exists an edge $\epsilon$ in the grid $g$ such that $\epsilon \subseteq P_{v}$ for all $v \in K$, we say that $K$ is represented as an edge clique. Another way to represent $K$ in a $B_{1}$-EPG representation of $G$ is as follows. Every path $P_{v}, v \in K$, has one of the following properties: (i) $P_{v}$ is a $\lrcorner$-path (resp. ᄀ-path) with bend point $(x, y)$; (ii) $P_{v}$ is a $\llcorner$-path (resp. $\ulcorner$-path) with bend point $(x, y)$; (iii) $P_{v}$ uses row $y$ and contains the grid point $(x, y)$. If there exists a path $P_{v}, v \in K$, of each of these three types, we say that $K$ is represented as a claw clique with horizontal basis $y$ and center $(x, y)$. See Fig. 1 for an example of an edge clique and one of a claw clique. We define in a similar way claw cliques with vertical basis.

In [13] the authors showed the following result.

Theorem 1. Let $\langle\mathcal{P}, g\rangle$ be a $B_{1}-E P G$ representation on a grid $g$ of a graph $G$. Every clique in $G$ corresponds to either an edge clique or a claw clique in $\langle\mathcal{P}, \mathcal{g}\rangle$.

Consider $H_{4}$ with edge set $\{a b, b c, c d, a d\}$ in a $B_{1}$-EPG graph $G$, and consider a $B_{1}$-EPG representation of $G$. If $P_{a}$ is a $\lrcorner$-path with bend point $(x, y), P_{b}$ is a $\left\llcorner\right.$-path with bend point $(x, y), P_{c}$ is a $\left\ulcorner\right.$-path with bend point $(x, y)$, and $P_{d}$ is a $\urcorner$-path with bend point $(x, y)$, then we say that $H_{4}$ is represented as a true pie with center $(x, y)$. Another possible representation of $H_{4}$ is as follows. $P_{a}$ is a $\lrcorner$-path (resp. $\left\llcorner\right.$-path) with bend point $(x, y), P_{b}$ uses column $x$ and contains $(x, y), P_{c}$ is a $\ulcorner$-path (resp. $\urcorner$-path) with bend point $(x, y)$, and $P_{d}$ uses row $y$ and contains $(x, y)$. Such a representation of $H_{4}$ is called a false pie with center $(x, y)$. Finally a third possible way to represent $H_{4}$ is to use what the authors in [13] call a frame. A frame is a rectangle in $g$ such that each corner is the bend for a different member of $P_{a}, P_{b}, P_{c}, P_{d}$, the subpaths $P_{a} \cap P_{b}, P_{b} \cap P_{c}, P_{c} \cap P_{d}, P_{d} \cap P_{a}$ are 

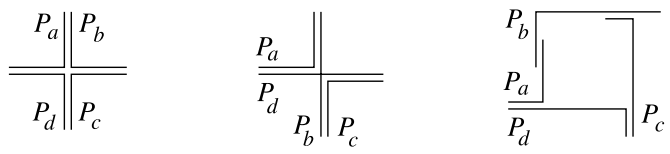

Fig. 2. $H_{4}$ represented as a true pie, a false pie, and a frame.
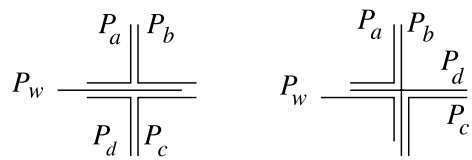

Fig. 3. Two possible $B_{1}$-EPG representations of $H_{4} \cup\{w\}$. Left: $H_{4}$ corresponds to a true pie. Right: $H_{4}$ corresponds to a false pie.

nonempty, and the subpaths $P_{b} \cap P_{d}, P_{a} \cap P_{c}$ are empty. See Fig. 2 for an example of a true pie, a false pie, and a frame. In [13] the authors showed the following result.

Theorem 2. Let $\langle\mathcal{P}, \mathcal{g}\rangle$ be a $B_{1}$-EPG representation on a grid $\mathcal{g}$ of a graph $G$. Every hole of length 4 in $G$ corresponds to a true pie or a false pie or a frame in $\langle\mathcal{P}, \mathcal{g}\rangle$.

\section{Some properties of the neighborhood}

In this section, we focus on the neighborhood of a vertex in a $B_{1}$-EPG graph. In particular we will give some properties of the subgraph induced by the neighborhood of any vertex in a $B_{1}$-EPG graph. First we start with an easy observation.

Lemma 3. Consider $H_{4}$ with edge set $\{a b, b c, c d, a d\}$ and let $w$ be adjacent to $a, b, c$ and $d$. Then in any $B_{1}$-EPG representation on a grid $g$ of $H_{4} \cup\{w\}, H_{4}$ corresponds either to a true pie or to a false pie.

Proof. From Theorem 2 it follows that in any $B_{1}$-EPG representation, $H_{4}$ corresponds to a true pie, or a false pie, or a frame. Suppose that $H_{4}$ corresponds to a frame. Thus every path $P_{i}, i \in V(H)$, is bended and two paths cannot have a same bend point. So we may assume, without loss of generality, that $P_{a}, P_{b}, P_{c}, P_{d}$ have bend points, respectively in $\left(x_{1}, y_{1}\right)$, $\left(x_{2}, y_{1}\right)$, $\left(x_{2}, y_{2}\right),\left(x_{1}, y_{2}\right)$. Now in order to intersect both paths $P_{a}$ and $P_{c}$, the path $P_{w}$ must be bended either in $\left(x_{1}, y_{2}\right)$ or in $\left(x_{2}, y_{1}\right)$. In the first case $P_{w}$ will clearly not intersect $P_{b}$ and in the second case $P_{w}$ will clearly not intersect $P_{d}$. Thus $H_{4}$ cannot be represented as a frame.

If $H_{4}$ corresponds to a true pie with center $(x, y)$, then $P_{w}$ intersects the paths of the pie either on column $x$ or on row $y$ (see Fig. 3). If $H_{4}$ corresponds to a false pie with center $(x, y)$, we may assume, without loss of generality, that $P_{a}$ is represented as a $\lrcorner$-path, $P_{b}$ uses column $x$ and contains $(x, y), P_{c}$ is represented as a $\left\ulcorner\right.$-path, and $P_{d}$ uses row $y$ and contains $(x, y)$. Then $P_{w}$ is either a $\llcorner$-path with bend point $(x, y)$ or a $\urcorner$-path with bend point $(x, y)$ (see Fig. 3 ).

Let us now focus on some configurations which can never occur in the neighborhood of a vertex in a $B_{1}$-EPG graph.

Lemma 4. Let $G$ be a $B_{1}$-EPG graph. Then $G \mid N(v)$ does not contain $\bar{H}_{6}, \bar{P}_{6}$ as induced subgraphs, for all $v \in V(G)$.

Proof. The proof is by contradiction. We will prove that $G \mid N(v)$ does not contain $\bar{H}_{6}$. The proof for $\bar{P}_{6}$ is similar. Consider $\bar{H}_{6}$ with vertex set $\{a, b, c, d, e, f\}$ and edge set $\{a b, b c, c d, a d$, ae, de, ef, $b f, c f\}$. Suppose a vertex $v \in V(G)$ is complete to $V\left(H_{6}\right)$. It follows from Lemma 3 that the hole $H_{4}$ with vertex set $a, b, c, d$ is represented either as a true pie or as a false pie. First suppose that $H_{4}$ is represented as a true pie with center $\left(x_{i}, y_{j}\right)$. Without loss of generality, we may assume that $P_{a}$ is represented as a $\lrcorner$-path, $P_{b}$ as a $\left\llcorner\right.$-path, $P_{c}$ as a $\left\ulcorner\right.$-path, and $P_{d}$ as a $\urcorner$-path. Since $e$ is adjacent to $a$ and $d$, but not to $b$ and $c, P_{e}$ must use row $y_{j}$ only at the left of column $x_{i}$ and cannot use column $x_{i}$. Similarly since $f$ is adjacent to $b$ and $c$, but not to $a$ and $d, P_{f}$ must use row $y_{j}$ only at the right of column $x_{i}$ and cannot use column $x_{i}$. But now clearly $P_{e}$ and $P_{f}$ cannot intersect, a contradiction (see Fig. 4). So suppose that $H_{4}$ is represented as a false pie with center $\left(x_{i}, y_{j}\right)$. Without loss of generality, we may assume that $P_{a}$ is represented as a $\lrcorner$-path, $P_{b}$ uses column $x_{i}$ and contains $\left(x_{i}, y_{j}\right), P_{c}$ is represented as a $\ulcorner$-path, and $P_{d}$ uses row $y_{j}$ and contains $\left(x_{i}, y_{j}\right)$. Using the same arguments as before we obtain that $P_{e}$ must use row $y_{j}$ only at the left of $x_{i}$ and cannot use column $x_{i}$, and $P_{f}$ must use column $x_{i}$ only below row $y_{j}$ and cannot use row $y_{j}$. Thus $P_{e}$ and $P_{f}$ cannot intersect, a contradiction (see Fig. 4).

Since every antihole of length at least 7 contains $\bar{P}_{6}$ as an induced subgraph, the following result follows immediately from Lemma 4.

Corollary 5. Let $G$ be a $B_{1}-E P G$ graph. Then $G \mid N(v)$ contains no $\bar{H}_{k}, k \geq 6$, for all $v \in V(G)$.

We prove the following lemma.

Lemma 6. Let $G$ be a $B_{1}$-EPG graph. Then $G \mid N(v)$ contains no $H_{k}, k \geq 5$, for all $v \in V(G)$. 

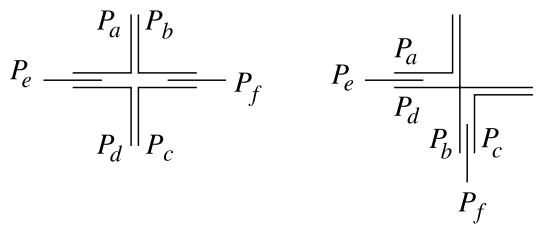

Fig. 4. Left: $H_{4}$ represented as a true pie; $P_{e}$ and $P_{f}$ cannot intersect. Right: $H_{4}$ represented as a false pie; $P_{e}$ and $P_{f}$ cannot intersect.

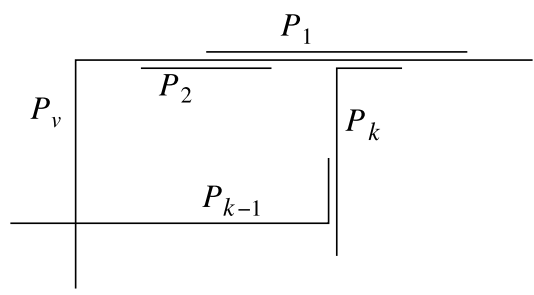

Fig. 5. $P_{k-1}$ cannot intersect $P_{k}$ and $P_{v}$.

Proof. The proof is by contradiction. Consider $H_{k}, k \geq 5$, with vertex set $\left\{a_{1}, a_{2}, \ldots, a_{k}\right\}$ and edge set $\left\{a_{1} a_{2}, a_{2} a_{3}, \ldots\right.$, $\left.a_{k-1} a_{k}, a_{k} a_{1}\right\}$. Suppose that a vertex $v \in V(G)$ is complete to $V\left(H_{k}\right)$. Without loss of generality, we may assume that $P_{v}$ is represented as a $\left\ulcorner\right.$-path with bend point $\left(x_{i}, y_{j}\right)$ and endpoints $\left(x_{i^{\prime}}, y_{j}\right),\left(x_{i}, y_{j^{\prime}}\right)$. Furthermore, since $k \geq 5$, we may assume that there are at least three paths representing vertices of $H_{k}$ which intersect $P_{v}$ on row $y_{j}$. Thus we may assume that $P_{a_{1}}$ is the path having the rightmost intersection with $P_{v}$ on row $y_{j}$ among the paths $P_{a_{i}}, i \in\{1, \ldots, k\}$. We may also assume that the intersection of $P_{a_{1}}$ with $P_{v}$ lies between columns $x_{l}$ and $x_{l^{\prime}}, i<l<l^{\prime} \leq i^{\prime}$. Since $P_{a_{2}}$ and $P_{a_{k}}$ do not intersect, we may assume that the intersection of $P_{a_{k}}$ with $P_{a_{1}}$ is at the right of the intersection of $P_{a_{2}}$ with $P_{a_{1}}$. This implies that $P_{a_{k}}$ intersects $P_{v}$ only on row $y_{j}$. Since $P_{a_{1}}$ is the path having the rightmost intersection with $P_{v}$ on row $y_{j}$, and since $P_{a_{k-1}}$ does not intersect either $P_{a_{1}}$ or $P_{a_{2}}$, it follows that $P_{a_{k-1}}$ must intersect $P_{a_{k}}$ on some column $x_{p}, l \leq p \leq l^{\prime}$, and $P_{a_{k-1}}$ must intersect $P_{v}$ on column $x_{i}$. But this is clearly impossible since $P_{a_{k-1}}$ has a single bend (see Fig. 5).

We can now state our main result of this section.

Theorem 7. Let $G$ be a $B_{1}-E P G$ graph. Then $G \mid N(v)$ is weakly chordal, for all $v \in V(G)$.

Proof. It follows from Lemma 6 that $G \mid N(v)$ contains no $H_{k}, k \geq 5$, and does not contain $\bar{H}_{5}$ (since $H_{5}$ is isomorphic to its complement), for all $v \in V(G)$. From Corollary 5 it follows that $G \mid N(v)$ contains no $\bar{H}_{k}, k \geq 6$, for all $v \in V(G)$. This proves the theorem.

Since weakly chordal graphs are perfect graphs, we obtain the following result.

Lemma 8. Let $G$ be a $B_{1}-E P G$ graph. Then $G \mid N(v)$ is perfect, for all $v \in V(G)$.

Finally let us show the following result which we will use later in the paper.

Theorem 9. Let $G$ be a $B_{1}-E P G$ graph. Then $G \mid N(v)$ is AT-free, for all $v \in V(G)$.

Proof. The proof is by contradiction. Suppose that $G \mid N(v)$ contains an asteroidal triple $a, b, c$ for some vertex $v \in V(G)$. Suppose, without loss of generality, that $P_{v}$ is represented as a $\left\ulcorner\right.$-path with bend point $\left(x_{i}, y_{j}\right)$ and endpoints $\left(x_{i}, y_{j^{\prime}}\right),\left(x_{i^{\prime}}, y_{j}\right)$. We may assume that $P_{a}$ and $P_{b}$ intersect $P_{v}$ on row $y_{j}$, that $P_{a}$ is at the right of $P_{b}$, and that $P_{c}$ intersects $P_{v}$ on row $y_{j}$ at the left of $P_{b}$ or on column $x_{i}$. Let $\left(x_{l}, y_{j}\right)$ be the rightmost intersection point of $P_{b}$ with $P_{v}, i<l<i^{\prime}$. In $G \mid N(v)$ there exists a path $\pi=\left\{a v_{1}, v_{1} v_{2}, \ldots, v_{q-1} v_{q}, v_{q} c\right\}$ from $a$ to $c$ avoiding the neighborhood of $b$. Clearly there exists at least one vertex $v_{i}, i \in\{1, \ldots, q\}$, such that the corresponding path $P_{v_{i}}$ contains a grid point lying at the left of column $x_{l}$. Let $v_{p}$ be the first vertex in the order $\left\{v_{1}, v_{2}, \ldots, v_{q}\right\}$ whose corresponding path contains a grid point lying at the left of column $x_{l} . P_{v_{p}}$ cannot contain a grid point on row $y_{j}$ at the left of column $x_{l}$, otherwise it intersects $P_{b}$. Thus $P_{v_{p}}$ uses some row $y_{j^{\prime \prime}}, j^{\prime \prime} \neq j$ (see Fig. 6). Since $P_{v_{p}}$ must intersect $P_{v}$, it necessarily uses column $x_{i}$. Now consider $P_{v_{p-1}}$. By definition of $v_{p}, P_{v_{p-1}}$ lies at the right of column $x_{l}$ and must intersect $P_{v_{p}}$ on row $y_{j^{\prime \prime}}$. But then clearly $P_{v_{p-1}}$ cannot intersect $P_{v}$, since they must necessarily intersect on row $y_{j}$.

As a consequence we get the following result.

Corollary 10. Let $G$ be a $B_{1}-E P G$ graph not containing $H_{4}$. Then for every vertex $v \in V(G), G \mid N(v)$ is an interval graph.

Proof. Let $v \in V(G)$. Since $G$ does not contain $H_{4}$ and because $G \mid N(v)$ cannot contain $H_{k}, k \geq 5$ (see Lemma 6 ), $G \mid N(v)$ is a chordal graph. From Theorem 9, we know that $G \mid N(v)$ is AT-free. By a result from [15], a graph is an interval graph if and only if it is chordal and AT-free. Therefore $G \mid N(v)$ is an interval graph. 


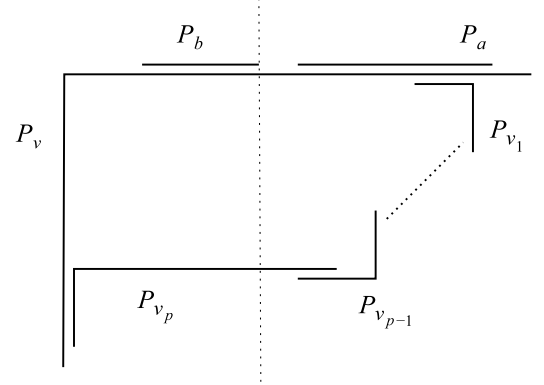

Fig. 6. Illustrating the proof of Theorem 9.

\section{The Erdős-Hajnal property for $B_{1}$-EPG graphs}

In [10], Erdős and Hajnal made the following conjecture.

Conjecture 11. For every graph $H$, there exists $\delta(H)>0$ such that if $G$ is a graph and no induced subgraph of $G$ is isomorphic to $H$, then $G$ contains either a clique or a stable set of size at least $|V(G)|^{\delta(H)}$.

Related to this, we consider the following definition (see [12]).

Definition 12. A family $\mathcal{F}$ of finite graphs has the Erdős-Hajnal property if there exists a constant $\epsilon(\mathcal{F})>0$ such that every graph in $\mathcal{F}$ on $n$ vertices contains either a clique or a stable set of size at least $n^{\epsilon(\mathcal{F})}$.

Notice that the family $\mathcal{F}$ of perfect graphs satisfies the Erdős-Hajnal property with $\epsilon(\mathcal{F})=\frac{1}{2}$. Indeed for any perfect graph $G$ on $n$ vertices we have $n \leq \omega(G) \alpha(G)$, where $\omega(G)$ is the size of a largest clique in $G$ and $\alpha(G)$ is the size of a largest stable set in $G$. It follows that either $\omega(G)$ or $\alpha(G)$ has value at least $n^{\frac{1}{2}}$.

It follows from $[1,12]$ that the family $\mathcal{F}$ of $B_{1}$-EPG graphs satisfies the Erdős-Hajnal property but without giving any fixed value of $\epsilon(\mathcal{F})$. Here we will prove that the family $\mathcal{F}$ of $B_{1}$-EPG graphs satisfy the property with $\epsilon(\mathcal{F})=\frac{1}{3}$.

Theorem 13. Let $G=(V, E)$ be a $B_{1}-E P G$ graph with $|V|=n$. Then $G$ contains either a clique or a stable set of size at least $n^{\frac{1}{3}}$.

Proof. First suppose that there exists a vertex $v \in V(G)$ with degree at least $n^{\frac{2}{3}}$. Thus it follows from Lemma 8 that $G \mid N(v)$ is a perfect graph of size at least $n^{\frac{2}{3}}$. From the remark above we deduce that $G$ contains either a clique or a stable set of size $\left(n^{\frac{2}{3}}\right)^{\frac{1}{2}}=n^{\frac{1}{3}}$.

Now suppose that $G$ does not contain any vertex with degree at least $n^{\frac{2}{3}}$. Thus $G$ has maximum degree at most $n^{\frac{2}{3}}-1$. In [5] it was proven that every maximal stable set in a graph on $n$ vertices and of maximum degree $h$ has size at least $\left\lceil\frac{n}{h+1}\right\rceil$. It follows that in our case $G$ contains a stable set of size at least $\left\lceil\frac{n}{\left(n^{\frac{2}{3}}-1\right)+1}\right\rceil \geq n^{\frac{1}{3}}$.

\section{Subclasses of chordal graphs}

In this section we focus on subclasses of chordal graphs. We consider several classes of chordal graphs: chordal bullfree graphs, chordal claw-free graphs, chordal diamond-free graphs (see Fig. 7; formal definitions will be given in the corresponding sections), and finally special cases of split graphs. We give necessary and sufficient conditions for such graphs to be $B_{1}$-EPG graphs or we prove that all graphs of the class considered are $B_{1}$-EPG.

We start with two easy observations.

Lemma 14. Let $G$ be a $B_{1}-E P G$ graph and let $K$ be a maximal clique in $G$.

(1) If $K$ is represented as an edge clique, then there exists a segment on the grid which is used by all paths corresponding to the vertices of $K$ and no other path uses that segment.

(2) If $K$ is represented as a claw clique with a horizontal basis and with center $C=\left(x_{i}, y_{j}\right)$ and if $G$ is $C_{4}$-free, then there exists $a B_{1}-E P G$ representation of $G$ in which all the paths corresponding to the vertices of $K$ use row $y_{j}$ between column $x_{i-1}$ and column $x_{i+1}$ and no other path uses that segment.

Proof. (1) By definition of an edge clique there exists an edge on the grid such that all paths corresponding to vertices of $K$ use this edge. If there is a path $P_{v}$ with $v \notin K$ using this same edge, then $K$ is not maximal, a contradiction. This proves (1). 


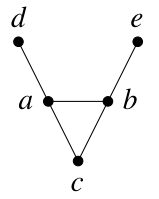

bull

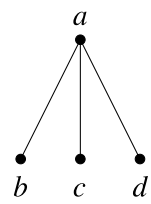

claw

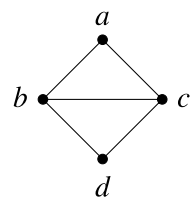

diamond

Fig. 7. Bull, claw, and diamond graphs.

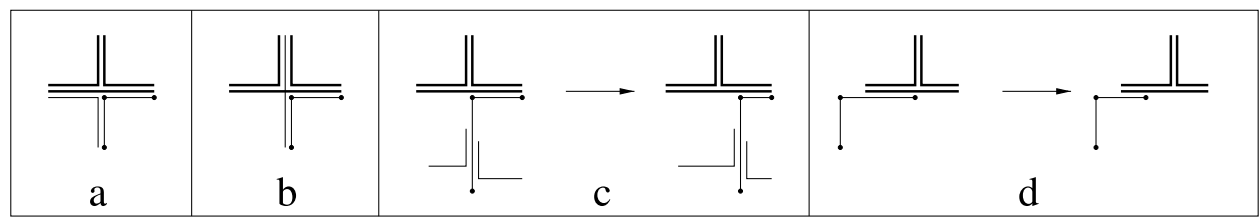

Fig. 8. In this figure, $P_{v}$ is represented by a path with small dots as endpoints and the bend point. (a) $P_{v}$ intersects a ?-path with bend point $C$. (b) The vertical segment of $P_{v}$ intersects a path using the grid segment $\left\{x_{1}\right\} \times\left[y_{0}, y_{1}\right]$. (c) The vertical segment of $P_{v}$ intersects a path not containing $C$. (d) $C$ is an endpoint of $P_{v}$.

(2) Denote by $\mathcal{K}$ the set of paths corresponding to vertices of $K$. Assume, without loss of generality, that the basis of the claw clique is $\left[x_{0}, x_{2}\right] \times\left\{y_{0}\right\}$ and that $C=\left(x_{1}, y_{0}\right)$. If no path $P_{v} \notin \mathcal{K}$ uses row $y_{0}$ between columns $x_{0}$ and $x_{2}$, then we are done. So we may assume now that there exists a path $P_{v} \notin \mathcal{K}$ such that $P_{v}$ uses the grid segment $\left[x_{1}, x_{2}\right] \times\left\{y_{0}\right\}$. Clearly $P_{v}$ cannot use either the segment $\left[x_{0}, x_{1}\right] \times\left\{y_{0}\right\}$ or the segment $\left\{x_{1}\right\} \times\left[y_{0}, y_{1}\right]$, otherwise $v \in K$, a contradiction. Thus we may distinguish two cases: (i) $C$ is an endpoint of $P_{v}$; (ii) $P_{v}$ is a $\ulcorner$-path with bend point $C$.

First we transform the grid $g$ into a new grid $g^{\prime}$ by inserting between every two columns $x_{i}, x_{i+1}$ a new column $x_{i^{\prime}}$. Thus in the new grid $g^{\prime}$ all paths $P$ have doubled in length as regards their horizontal part.

If $C$ is an endpoint of $P_{v}$, then we may shorten $P$ on row $y_{0}$ without changing the adjacencies, i.e., we may delete the horizontal part of $P_{v}$ using the segment $\left[x_{1}, x_{2}\right] \times\left\{y_{0}\right\}$ such that $\left(x_{1^{\prime}}, y_{0}\right)$ becomes a new endpoint of $P_{v}$ (see Fig. 8(d) for an example).

So we may assume now that $P_{v}$ is a $\ulcorner$-path with bend point $C$. Then we distinguish three cases: (i) the vertical segment of $P_{v}$ intersects a $\urcorner$-path with bend point $C$ (see Fig. 8(a)); (ii) the vertical segment of $P_{v}$ intersects a path using the grid segment $\left\{x_{1}\right\} \times\left[y_{0}, y_{1}\right]$ (see Fig. 8(b)); (iii) the vertical segment of $P_{v}$ intersects a path not containing $C$ (see Fig. 8(c)). Clearly in the first two cases we get a $C_{4}$ represented as a true pie in case (i) and represented as a false pie in case (ii), a contradiction. In case (iii) we may transform the representation so that all the paths using $x_{1}$ and not contained in $\mathcal{K}$ will use instead column $x_{1^{\prime}}$. To do so, we have to extend or shorten these paths in order to keep the same adjacencies (see Fig. 8(c)).

Note that similar transformations are applied if $P_{v}$ is a path using the grid segment $\left[x_{0}, x_{1}\right] \times\left\{y_{0}\right\}$ in the original grid $g$. Thus we obtain a representation in which only paths corresponding to vertices in $K$ use the grid segment $\left[x_{0^{\prime}}, x_{1^{\prime}}\right] \times\left\{y_{0}\right\}$.

Notice that Lemma 14(2) can also be stated with the claw clique having a vertical basis.

\subsection{Chordal bull-free graphs}

A bull is a graph with vertex set $\{a, b, c, d, e\}$ and with edge set $\{a b, a c, a d, b c, b e\}$. A graph is bull-free if it does not contain any induced subgraph isomorphic to a bull.

A homogeneous set in a graph $G$ is a subset $H \subseteq V(G)$ with $2 \leq|H|<|V(G)|$, such that every vertex in $G \backslash H$ is either complete to $H$ or anticomplete to $H$. For such an $H$, let $N(H)$ denote the set of vertices in $G \backslash H$ which are complete to $H$ and $\bar{N}(H)$ the set of vertices in $G \backslash H$ which are anticomplete to $H$.

In [16], the authors show that every connected bull-free graph $G$ satisfies one of the following five properties: (i) $G$ contains $H_{5}$, (ii) $G$ is triangle-free, (iii) $\bar{G}$ is triangle-free, (iv) $G$ has a homogeneous set, (v) $G$ or $\bar{G}$ contains an induced subgraph isomorphic to $F_{0}=\{a b, b c, c d, a d, a e, b e, c f\}$. This result will be used in order to prove the main result of this subsection.

We will first start with the following observation.

Lemma 15. Let $G$ be a $\left\{\right.$ bull, $\left.C_{4}\right\}$-free $B_{1}$-EPG graph. Then $G$ admits a $B_{1}$-EPG representation in which every clique is represented as an edge clique.

Proof. Suppose the result is false, i.e., for every $B_{1}$-EPG representation of $G$ there exists at least one clique in $G$ which is represented as a claw clique. Consider one such representation and let $K$ denote a clique in $G$ which is represented as a claw clique. Without loss of generality, we may assume that $K$ is maximal, and that the claw clique has horizontal basis $\left[x_{0}, x_{2}\right] \times\left\{y_{0}\right\}$ and center $C=\left(x_{1}, y_{0}\right)$. From Lemma 14(2) it follows that we may assume that no path $P_{v}, v \notin K$, uses the grid segment $\left[x_{0}, x_{2}\right] \times\left\{y_{0}\right\}$. Let us denote by $\mathcal{K}$ the set of paths corresponding to the vertices of $K$. For every $\lrcorner$-path $P_{v} \in \mathcal{K}$ 
(resp. $\left\llcorner\right.$-path $P_{v^{\prime}} \in \mathcal{K}$ ), we do the following: if $P_{v}$ (resp. $P_{v^{\prime}}$ ) does not intersect any path $P_{w} \notin \mathcal{K}$ on column $x_{1}$, then we delete its vertical segment and add the grid segment $\left[x_{1}, x_{2}\right] \times\left\{y_{0}\right\}$ (resp. $\left.\left[x_{0}, x_{2}\right] \times\left\{y_{0}\right\}\right)$. If after these transformations either there exist no more $\lrcorner$-paths in $\mathcal{K}$ or there exist no more $\llcorner$-paths in $\mathcal{K}$, then we are done since we have obtained an edge clique. So we may assume that there exists at least one $\lrcorner$-path $P_{v} \in \mathcal{K}$ and at least one $\left\llcorner\right.$-path $P_{v^{\prime}} \in \mathcal{K}$. Since both paths $P_{v}, P_{v^{\prime}}$ intersect a path on column $x_{1}$, they necessarily intersect a common path, say $P_{t} \notin \mathcal{K}$. Now if all $\lrcorner$-paths (resp. $\llcorner$-paths) do not intersect any path on row $y_{0}$, then we may transform them into ᄂ-paths (resp. $\lrcorner$-paths) with rightmost endpoint $\left(x_{2}, y_{0}\right)$ (resp. with leftmost endpoint $\left.\left(x_{0}, y_{0}\right)\right)$ and thus obtain an edge clique. So we may assume that there exists at least one $\lrcorner$-path intersecting a path $P_{t^{\prime}} \notin \mathcal{K}$ on row $y_{0}$ and there exists at least one $\left\llcorner\right.$-path intersecting a path $P_{t^{\prime \prime}} \notin \mathcal{K}$ on row $y_{0}$. Without loss of generality, we may assume that $P_{v}$ intersects $P_{t^{\prime}}$ and that $P_{v^{\prime}}$ intersects $P_{t^{\prime \prime}}$. Clearly $P_{t^{\prime}}$ and $P_{t^{\prime \prime}}$ cannot intersect since $t^{\prime}, t^{\prime \prime} \notin K$ and all paths are single-bended. By the same arguments, $P_{t^{\prime}}, P_{t^{\prime \prime}}$ cannot intersect $P_{t}$. But now $v, v^{\prime}, t, t^{\prime}, t^{\prime \prime}$ induce a bull, a contradiction.

We are now in a position to prove our main result of this subsection.

Theorem 16. Let $G$ be a chordal bull-free graph. Then $G$ is a $B_{1}-E P G$ graph if and only if it does not contain any induced subgraph isomorphic to $T_{2}=\{a b, b c, c d, d e, c f, f g\}$ in the neighborhood of a vertex.

Proof. From Theorem 9 it follows that if $G$ is a $B_{1}$-EPG graph then $T_{2}$ is a forbidden induced subgraph for $G \mid N(v), \forall v \in V(G)$, since $a, e$ and $g$ form an asteroidal triple. For the "if" direction, assume that the result is false and consider a minimal counterexample $G^{\prime}$. Clearly $G^{\prime}$ must be connected. If $G^{\prime}$ is AT-free, then $G^{\prime}$ is an interval graph (see [15]) and thus it is $B_{1}$-EPG, a contradiction. So suppose that $G^{\prime}$ contains an asteroidal triple. Since $G^{\prime}$ is chordal and bull-free, it must contain $T_{2}$ as an induced subgraph. Indeed, $T_{2}$ is the only graph among the forbidden induced subgraphs that characterize AT-free graphs which does not contain either a hole or a bull (see [8]). From the aforementioned result [16], it follows that $G^{\prime}$ must contain a homogeneous set $H$. Indeed, $G^{\prime}$ does not contain $H_{5}$ since it is chordal; $G^{\prime}$ is not triangle-free since otherwise it is a tree and thus $B_{1}$-EPG (see [13]); $\bar{G}^{\prime}$ is not triangle-free since $G^{\prime}$ must contain $T_{2}$ as an induced subgraph $\left(b, d, f\right.$ induce a triangle in $\left.\overline{G^{\prime}}\right)$; neither $G^{\prime}$ nor $\overline{G^{\prime}}$ contains $F_{0}$ as an induced subgraph since both $F_{0}$ and $\bar{F}_{0}$ contain $H_{4}$. Therefore $G^{\prime}$ must satisfy the remaining property, i.e., $G^{\prime}$ must contain a homogeneous set $H$. Since $|H|<|V(G)|$ and $G^{\prime}$ is connected, we must have $|N(H)| \geq 1$. Next notice that $H$ does not contain $T_{2}$ as an induced subgraph, since otherwise $T_{2}$ is in the neighborhood of every vertex of $N(H)$. Thus $G^{\prime} \mid H$ is an interval graph since it is chordal and AT-free. Furthermore either $G^{\prime} \mid H$ or $G^{\prime} \mid N(H)$ is a clique. Indeed, if neither of them is a clique, i.e., if there exist two vertices $v_{1}, v_{2} \in H$ and two vertices $w_{1}, w_{2} \in N(H)$ such that $v_{1} v_{2}, w_{1} w_{2} \notin E\left(G^{\prime}\right)$, then these vertices induce a $C_{4}$, a contradiction. First suppose that $G^{\prime} \mid H$ is a clique. Delete $v_{1} \in H$. $G^{\prime} \backslash\left\{v_{1}\right\}$ admits a $B_{1}$-EPG representation by minimality of $G^{\prime}$. Now add $P_{v_{1}}$ to that representation such that $P_{v_{1}}$ coincides with $P_{v_{2}}$ for some $v_{2} \in H$ ( $v_{2}$ exists since $|H| \geq 2$ ). This clearly gives us a feasible $B_{1}$-EPG representation of $G^{\prime}$, a contradiction. So suppose $G^{\prime} \mid N(H)$ is a clique. Consider a $B_{1}$-EPG representation of $G^{\prime \prime}=G^{\prime} \mid(V \backslash H) \cup\left\{v^{\prime}\right\}$ where $v^{\prime} \in H$ ( $v^{\prime}$ exists since $|H| \geq 2$ ). It follows from Lemma 15 that we may assume that the clique $K$ induced by $N(H) \cup\left\{v^{\prime}\right\}$ is represented as an edge clique. Since $K$ is maximal in $G^{\prime \prime}$, it follows from Lemma 14 that we may assume that only paths representing vertices of $K$ use row $y_{0}$ between columns $x_{i}$ and $x_{j}, i<j$. Now delete $P_{v^{\prime}}$ and add a $B_{1}$-EPG representation of $G^{\prime} \mid H$ on row $y_{0}$ between columns $x_{i}$ and $x_{j}$. This is possible because $G^{\prime} \mid H$ is an interval graph and we may choose $x_{i}, x_{j}$ such that a $B_{1}$-EPG representation of $G^{\prime} \mid H$ fits between these columns on row $y_{0}$. Thus we get a $B_{1}$-EPG representation of $G^{\prime}$, a contradiction.

\subsection{Chordal claw-free graphs}

A claw is a graph with vertex set $\{a, b, c, d\}$ and edge set $\{a b, a c, a d\}$. A graph is claw-free if it does not contain any induced subgraph isomorphic to the claw. A claw with vertices $a, b, c, d$ and edges $a b, a c, a d$ will be denoted by $(a ; b, c, d)$. A simplicial vertex in a graph is a vertex whose neighbors induce a clique. From [9], we know that every chordal graph has a simplicial vertex.

\section{Theorem 17. Every chordal claw-free graph $G$ is a $B_{1}-E P G$ graph.}

Proof. Suppose that the statement is wrong. Then let $G$ be a minimal counterexample, i.e., let $G$ be a chordal claw-free graph such that $G$ has no $B_{1}$-EPG representation, but $G \backslash\{v\}$ has a $B_{1}$-EPG representation for every vertex $v \in V(G)$. We will consider several cases and in all of them we will deduce that $G$ actually has a $B_{1}$-EPG representation; thus we get a contradiction.

According to the result mentioned above from [9], $G$ has a simplicial vertex. Let $v$ be such a vertex. Let $G^{\prime}=G \backslash\{v\}$ and let $\left\langle\mathcal{P}^{\prime}, g\right\rangle$ be a $B_{1}$-EPG representation of $G^{\prime}$. Let $K$ be the clique induced by $N(v)$ and denote by $\mathcal{K}$ the set of paths in $\left\langle\mathcal{P}^{\prime}, g\right\rangle$ corresponding to the vertices in $K$. If $K$ is a maximal clique in $G^{\prime}$, then it follows from Lemma 14 that there exists a segment on the grid used by all the paths in $\mathcal{K}$ and no other path uses this segment. Thus we may add a path $P_{v}$ on this segment and hence we obtain a $B_{1}$-EPG representation of $G$, a contradiction.

Therefore we may assume that $K$ is not maximal. Thus there exists at least one vertex $w \in V\left(G^{\prime}\right)$ which is complete to $K$. Let $W$ be the set of all such vertices $w$ and denote by $W$ the set of paths in $\left\langle\mathcal{P}^{\prime}, g\right\rangle$ corresponding to the vertices in $W$. We make the following easy observations:

Observation I. If $w_{1}, w_{2} \in W$, then $w_{1} w_{2} \in E(G)$. Indeed, if $w_{1} w_{2} \notin E(G)$, then $\left(u ; v, w_{1}, w_{2}\right)$ is a claw for any $u \in K$, a contradiction. 
Observation II. If $u t \in E(G)$, for $u \in K$ and $t \notin W \cup K \cup\{v\}$, then $w t \in E(G)$ for all $w \in W$. Indeed, if $w t \notin E(G)$, then $(u ; v, t, w)$ is a claw, a contradiction.

Observation III. If $u t_{1}, u t_{2} \in E(G)$ for $u \in K$ and $t_{1}, t_{2} \notin W \cup K \cup\{v\}$, then $t_{1} t_{2} \in E(G)$. Indeed, if $t_{1} t_{2} \notin E(G)$, then $\left(u ; v, t_{1}, t_{2}\right)$ is a claw, a contradiction.

It follows from Observation I that $K \cup W$ is a clique-the unique maximal clique including $K$ in $G^{\prime}$. We will distinguish two cases according to whether $K \cup W$ is represented in $\left\langle\mathcal{P}^{\prime}, \mathscr{g}\right\rangle$ by an edge clique or by a claw clique.

Case 1: $K \cup W$ is represented in $\left\langle\mathcal{P}^{\prime}, g\right\rangle$ by an edge clique.

It follows from Lemma 14 that we may assume, without loss of generality, that the segment $\left[x_{0}, x_{2}\right] \times\left\{y_{0}\right\}$ is used by all paths of $\mathcal{K} \cup \mathcal{W}$ and no other path uses that segment. From this and from the fact that all paths have at most one bend we obtain the following observation.

Observation IV. If a path $P_{t}$ intersects (on at least one edge) a path from $\mathcal{K} \cup \mathcal{W}$ and $t \notin K \cup W$, then the intersection lies either at the left of $x_{0}$ or at the right of $x_{2}$.

This observation allows us to define the following subsets of $V\left(G^{\prime}\right)$.

$T_{\ell}$ is the set of vertices $t \notin K \cup W$ such that $P_{t}$ intersects (on at least one edge) a path of $\mathcal{K}$ at the left of $x_{0}$;

$T_{r}$ is the set of vertices $t \notin K \cup W$ such that $P_{t}$ intersects (on at least one edge) a path of $\mathcal{K}$ at the right of $x_{2}$;

$K_{\ell}$ is the set of vertices $u \in K$ with at least one neighbor in $T_{\ell}$;

$K_{r}$ is the set of vertices $u \in K$ with at least one neighbor in $T_{r}$;

$T_{1}$ is the set of vertices $t \notin K \cup W \cup T_{\ell} \cup T_{r}$ such that $P_{t}$ intersects (on at least one edge) a path of $W$ at the left of $x_{0}$; $T_{2}$ is the set of vertices $t \notin K \cup W \cup T_{\ell} \cup T_{r}$ such that $P_{t}$ intersects (on at least one edge) a path of $W$ at the right of $x_{2}$.

In what follows we will prove several claims.

Claim 1. $W$ is complete to $T_{\ell} \cup T_{r}$.

This immediately follows from Observation II.

Claim 2. $T_{\ell} \cup T_{1}$ is anticomplete to $T_{r} \cup T_{2}$.

This follows from the fact that no path $P_{t}$ with $t \in T_{\ell} \cup T_{r} \cup T_{1} \cup T_{2}$ uses row $y_{0}$ between columns $x_{0}$ and $x_{1}$ and from the fact that all paths have at most one bend.

Claim 3. $K_{\ell} \cap K_{r}=\emptyset$.

Suppose that $u \in K_{\ell} \cap K_{r}$. Let $t \in T_{\ell}$ and $t^{\prime} \in T_{r}$ be two neighbors of $u$. It follows from Claim 2 that $t$ and $t^{\prime}$ are nonadjacent. But now $\left(u ; v, t, t^{\prime}\right)$ is a claw, a contradiction. This proves Claim 3.

Claim 4. $T_{\ell} \cup T_{1}, T_{r} \cup T_{2} \neq \emptyset$.

By symmetry it is enough to prove the claim for $T_{\ell} \cup T_{1}$. Suppose that $T_{\ell} \cup T_{1}=\varnothing$. Then we may assume that all paths in $\mathcal{K} \cup \mathcal{W}$ have their leftmost endpoint in $\left(x_{0}, y_{0}\right)$. Furthermore we may assume, without loss of generality, that no path is using the grid segment $\left[x_{-1}, x_{0}\right] \times\left\{y_{0}\right\}$. Thus we may extend the paths of $\mathcal{K}$ to the grid point $\left(x_{-1}, y_{0}\right)$ and then add $P_{v}$ as a path using only segment $\left[x_{-1}, x_{0}\right] \times\left\{y_{0}\right\}$. Hence we obtain a $B_{1}$-EPG representation of $G$, a contradiction. This proves Claim 4.

Claim 5. $K_{\ell}, K_{r} \neq \emptyset$.

If $K_{\ell}, K_{r}=\varnothing$, then it follows from Observation IV that no vertex in $K$ has a neighbor outside of $K \cup W \cup\{v\}$. Thus we may assume that all paths in $\mathcal{K}$ only use the segment $\left[x_{0}, x_{2}\right] \times\left\{y_{0}\right\}$. Without loss of generality, we may assume that no path uses the segment $\left\{x_{0}\right\} \times\left[y_{0}, y_{1}\right]$. Now we may add a vertical part $\left\{x_{0}\right\} \times\left[y_{0}, y_{1}\right]$ to all the paths in $\mathcal{K}$ and add $P_{v}$ as a path using only this segment, $\left\{x_{0}\right\} \times\left[y_{0}, y_{1}\right]$. Hence we obtain a $B_{1}$-EPG representation of $G$, a contradiction.

By symmetry we may assume that $K_{\ell} \neq \varnothing$. Let $u \in K_{\ell}$ and let $t \in T_{\ell}$ be a neighbor of $u$. Assume that $K_{r}=\emptyset$. This clearly implies that $T_{r}=\emptyset$. It follows from Claim 4 that $T_{2} \neq \emptyset$. Let $t_{2} \in T_{2}$ and let $w \in W$ be a neighbor of $t_{2}$. It follows from Observation II that $w t \in E(G)$ and it follows from Claim 2 that $t t_{2} \notin E(G)$. Furthermore it follows from Claim 3 that $u$ is nonadjacent to $T_{2}$. Since $t \notin K \cup W$, we conclude (by Observation II) that there exists a vertex $u^{*} \in K$ which is nonadjacent to $t$. It follows that $u^{*} t_{2} \in E(G)$, since otherwise $\left(w ; t, u^{*}, t_{2}\right)$ is a claw, a contradiction. But this implies that $P_{u^{*}}$ intersects $P_{t_{2}}$ necessarily at the right of $x_{2}$, and hence $u^{*} \in K_{r}$, a contradiction. This proves Claim 5 .

It follows from Claim 5 that $T_{\ell}, T_{r} \neq \emptyset$.

Claim 6. $T_{1}, T_{2}=\emptyset$.

By symmetry it is enough to show that $T_{1}=\emptyset$. Suppose that there exists a vertex $t_{1} \in T_{1}$ and let $w \in W$ such that $w t_{1} \in E(G)$. Consider $u \in K_{\ell}$. Since $t_{1} \notin T_{\ell}$, it follows that $u t_{1} \notin E(G)$. But now $\left(w ; u, t_{1}, t^{\prime}\right)$ is a claw for any vertex $t^{\prime} \in T_{r}$ (recall that $w t^{\prime} \in E(G)$ by Observation II and that $t_{1}, t^{\prime}$ are nonadjacent due to Claim 2 ), a contradiction. Thus $T_{1}=\emptyset$. This proves Claim 6. 


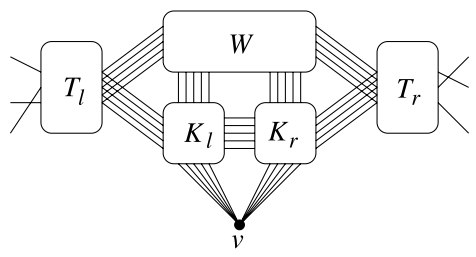

Fig. 9. The different sets of vertices and their relation.

Claim 7. $K_{\ell}$ is complete to $T_{\ell}$ and $K_{r}$ is complete to $T_{r}$.

By symmetry it is enough to show that $K_{\ell}$ is complete to $T_{\ell}$. Suppose $u \in K_{\ell}$ is nonadjacent to some vertex $t \in T_{\ell}$. Let $w \in W$ such that $w t^{\prime} \in E(G)$ for some $t^{\prime} \in T_{r} \cup T_{2}$ (Claim 4 implies that such a vertex $t^{\prime}$ exists). It follows from Claim 1 that $w t \in E(G)$ and it follows from Claim 2 that $t$ and $t^{\prime}$ are nonadjacent. Furthermore it follows from Claim 3 that $u$ is nonadjacent to $t^{\prime}$. But now $\left(w ; u^{\prime}, t, t^{\prime}\right)$ is a claw, a contradiction. This proves Claim 7.

Claim 8. $T_{\ell}$ and $T_{r}$ are cliques.

This immediately follows from Claim 7 and Observation III.

Claim 9. $K_{\ell} \cup K_{r}=K$.

Let $u \in K \backslash\left(K_{\ell} \cup K_{r}\right)$ and let $t \in T_{\ell}, t^{\prime} \in T_{r}, w \in W$. Then $t$ is nonadjacent to $t^{\prime}$ (see Claim 2) and $w$ is adjacent to both $t$ and $t^{\prime}$ (see Claim 1). Thus ( $\left.w ; u, t, t^{\prime}\right)$ is a claw, a contradiction. This proves Claim 8.

Fig. 9 illustrates the different sets of vertices and their relation.

From the above it follows that for each $u^{\prime} \in K_{\ell}$, we have $N_{G^{\prime}}[u]=K \cup W \cup T_{\ell}$, and that for each $u \in K_{r}$, we have $N_{G^{\prime}}[u]=K \cup W \cup T_{r}$. Therefore all the vertices in $K_{\ell}$ have exactly the same neighbors and all the vertices in $K_{r}$ have exactly the same neighbors.

It follows that we may assume that $\left|K_{\ell}\right|=\left|K_{r}\right|=1$. Indeed if for instance $u_{1}, u_{2} \in K_{\ell}$, then we obtain a $B_{1}$-EPG representation of $G$ by adding $P_{u_{2}}$ coinciding with $P_{u_{1}}$ to a $B_{1}$-EPG representation of $G \backslash\left\{u_{2}\right\}$. Thus we assume from now on that $K_{\ell}=\left\{u^{\prime}\right\}, K_{r}=\left\{u^{\prime \prime}\right\}$.

It also follows that for each $w \in W, N_{G^{\prime}}[w]=K \cup W \cup T_{\ell} \cup T_{r}$. Thus all the vertices in $W$ have exactly the same neighbors. Similarly to the assumption we did above, we may assume that $|W|=1$ and $W=\{w\}$.

Notice that at least one of the paths $P_{u^{\prime}}, P_{u^{\prime \prime}}$ is bended. For suppose not. We may assume that $P_{u^{\prime}}$ has its rightmost endpoint in $\left(x_{2}, y_{0}\right)$ and $P_{u^{\prime \prime}}$ has its leftmost endpoint in $\left(x_{0}, y_{0}\right)$. Without loss of generality, we may assume that no path uses the segment $\left\{x_{1}\right\} \times\left[y_{0}, y_{1}\right]$. Now we may bend $P_{u^{\prime}}$ and $P_{u^{\prime \prime}}$ at $\left(x_{1}, y_{0}\right)$ such that they will intersect along the segment $\left\{x_{1}\right\} \times\left[y_{0}, y_{1}\right]$ by deleting the part $\left[x_{1}, x_{2}\right] \times\left\{y_{0}\right\}$ from $P_{u^{\prime}}$ and the part $\left[x_{0}, x_{1}\right] \times y_{0}$ from $P_{u^{\prime \prime}}$. Adding $P_{v}$ on exactly this segment gives us a $B_{1}$-EPG representation of $G$, a contradiction. So, without loss of generality, we may assume that $P_{u^{\prime}}$ is a $\llcorner-$ path with bend point $\left(x_{-1}, y_{0}\right)$. It follows that there exists $t \in T_{\ell}$ such that $u^{\prime} t \in E(G)$ and the intersection of $P_{u^{\prime}}$ and $P_{t}$ is on column $x_{-1}$, since otherwise $P_{u^{\prime}}$ does not need to be bended.

Clearly at the left of $x_{0}, P_{w}$ and $P_{u^{\prime}}$ intersect exactly the same paths (namely $P_{t^{\prime}}$ for all $t^{\prime} \in T_{\ell}$ ). Thus we may assume that $P_{w}$ coincides with $P_{u^{\prime}}$ at the left of $x_{0}$. In particular, they are bended at $\left(x_{-1}, y_{0}\right)$. Since at the right of $x_{2}, P_{w}$ and $P_{u^{\prime \prime}}$ intersect precisely the same paths, namely $P_{t^{\prime \prime}}$ for all $t^{\prime \prime} \in T_{r}$, we may assume that $P_{u^{\prime \prime}}$ is not bended and coincides with $P_{w}$.

Recall that $T_{\ell}$ is a clique (see Claim 8). Thus it is represented either by an edge clique or by a claw clique. Recall that at least one path $P_{t}, t \in T_{\ell}$, intersects $P_{u^{\prime}}$ on column $x_{-1}$. We distinguish several cases which are also shown in Fig. 10. The bold paths correspond to $P_{w}, P_{u^{\prime}}, P_{u^{\prime \prime}}$ and $P_{t^{\prime \prime}}$ for some $t^{\prime \prime} \in T_{r}$. Thin paths represent paths $P_{t}$ for $t \in T_{\ell}$. Dotted vertical lines are $x=x_{0}$ and $x=x_{2}$. For each case that may occur, the figure presents a transformation which yields a $B_{1}$-EPG representation of $G$.

a. $T_{\ell}$ is represented by an edge clique and the common segment of all the paths $P_{t}, t \in T_{\ell}$, is on column $x_{-1}$ (see Fig. 10(a)). Notice that we may assume, without loss of generality, that no path $P_{t}, t \in T_{\ell}$, uses the grid segment $\left[x_{-2}, x_{-1}\right] \times\left\{y_{0}\right\}$. If a path $P_{t}, t \in T_{\ell}$, is a $\left\ulcorner\right.$-path or a $\left\llcorner\right.$-path with bend point $\left(x_{-1}, y_{0}\right)$, then its horizontal segment may be deleted. Under these assumptions, we may transform $P_{u^{\prime}}$ into a $\lrcorner$-path with bend point $\left(x_{-1}, y_{0}\right)$ and leftmost endpoint $\left(x_{-2}, y_{0}\right)$. Next we extend $P_{u^{\prime \prime}}$ such that its left endpoint becomes $\left(x_{-2}, y_{0}\right)$. Now it is possible to add $P_{v}$ on the grid segment $\left[x_{-2}, x_{-1}\right] \times\left\{y_{0}\right\}$ and thus we obtain a $B_{1}$-EPG representation of $G$, a contradiction.

b. $T_{\ell}$ is represented by a claw clique with its center on column $x_{-1}$ but not $\left(x_{-1}, y_{0}\right)$ (see Fig. 10(b)). Notice that the claw clique can only have a vertical basis. The same transformation as in case a. will lead to a contradiction.

c. $T_{\ell}$ is represented by a claw clique with a vertical basis and center $\left(x_{-1}, y_{0}\right)$ (see Fig. $10(\mathrm{c})$ ). Then the horizontal segments of all the paths $P_{t}, t \in T_{\ell}$, which use row $y_{0}$ may be deleted and their vertical segments slightly extended to keep all the intersections, which gives us an edge clique for $T_{\ell}$. Then we are in case a. again and thus we obtain a contradiction.

d. $T_{\ell}$ is represented by a claw clique with a horizontal basis and center $\left(x_{-1}, y_{0}\right)$ (see Fig. $10(\mathrm{~d})$ ). We delete the vertical segment of $P_{u^{\prime}}$ and extend the horizontal segment slightly to the left. Notice that we may assume that no path except $P_{t}, t \in T_{\ell}$, uses the grid segment $\left[x_{-2}, x_{-1}\right] \times\left\{y_{0}\right\}$. Now $P_{u^{\prime}}$ and $P_{u^{\prime \prime}}$ are both not bended. Then we conclude by using the transformation mentioned above.

This completes the proof for the case where $K \cup W$ is represented as an edge clique. 
a

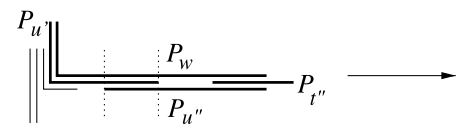

$\mathrm{b}$

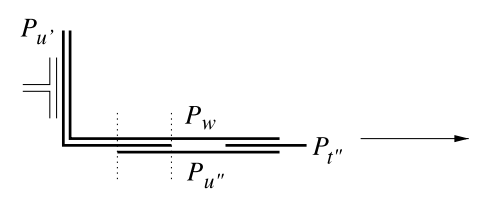

$\mathrm{C}$
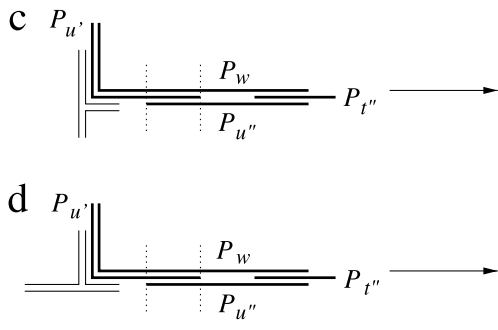
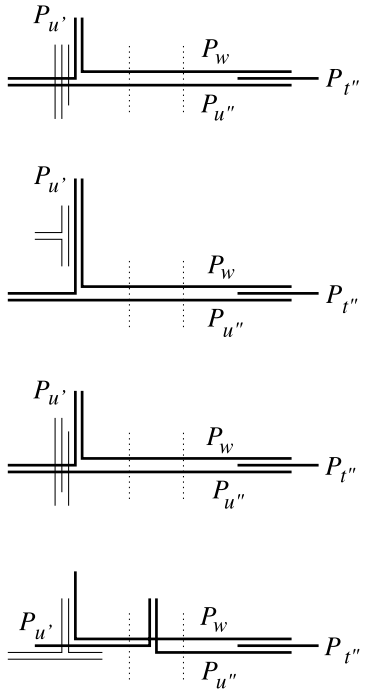

Fig. 10. Several cases that may occur and their transformation.

Case 2: $K \cup W$ is represented by a claw clique.

From Lemma 14 it follows that we may assume that the basis of the claw clique is on row $y_{0}$, the center is $\left(x_{1}, y_{0}\right)$, and the segments $\left[x_{0}, x_{2}\right] \times y_{0}$ and $x_{1} \times\left[y_{0}, y_{1}\right]$ are only used by paths corresponding to vertices in $K \cup W$.

First suppose there is no path $P_{t} \notin \mathcal{K} \cup \mathcal{W}$ which intersects a path $P_{u} \in \mathcal{K}$ on column $x_{1}$. Then we proceed as follows. If no $P_{w} \in \mathcal{W}$ uses column $x_{1}$, we can delete vertical segments on column $x_{1}$ of all the paths in $\mathcal{K}$ and extend their horizontal segments slightly (either to $\left(x_{2}, y_{0}\right)$ or to $\left.\left(x_{0}, y_{0}\right)\right)$ to preserve the intersections. Thus $K \cup W$ is represented as an edge clique and we are done. Notice that the same transformation can be applied in the case where all the paths $P_{w} \in \mathcal{W}$ which are bended at $\left(x_{1}, y_{0}\right)$ are $\lrcorner$-paths and in the case where all of them are $\llcorner$-paths.

The remaining case is when there are paths $P_{w}, P_{w^{\prime}} \in \mathcal{W}$ such that $P_{w}$ is a $\urcorner$-path and $P_{w^{\prime}}$ is a $\llcorner$-path. Then we may assume that all paths $P_{u} \in \mathcal{K}$ are not bended. Indeed, first notice that no $P_{u}$ can intersect some $P_{t} \notin \mathcal{K} \cup \mathcal{W}$ at the left of $x_{0}$ or at the right of $x_{2}$, otherwise $P_{w^{\prime}}$ (resp. $\left.P_{w}\right)$ cannot intersect $P_{t}$ (resp. $\left.P_{t^{\prime}}\right)$, thus contradicting Observation II. Furthermore if $P_{u}$ is a \lrcorner -path, then we may delete its vertical part and add the horizontal segment $\left[x_{1}, x_{2}\right] \times\left\{y_{0}\right\}$, and if $P_{u}$ is a $\llcorner$-path, then we may delete its vertical part and add the horizontal segment $\left[x_{0}, x_{1}\right] \times\left\{y_{0}\right\}$. Thus all the vertices $u \in K$ have the same neighbors, namely $W$. So we may assume that $|K|=1$, say $K=\{u\}$, and $P_{u}$ is not bended. Without loss of generality, we may assume that no path uses the segment on $\left\{x_{2}\right\} \times\left[y_{-1}, y_{0}\right]$. But now we can add to $P_{u}$ the vertical segment on $\left\{x_{2}\right\} \times\left[y_{-1}, y_{0}\right]$ and add $P_{v}$ on this segment to get a $B_{1}$-EPG representation of $G$, a contradiction.

Thus we may assume now that there exists $P_{t} \notin \mathcal{K} \cup \mathcal{W}$, which intersects a path $P_{u} \in \mathcal{K}$ on column $x_{1}$. Notice that there cannot exist $P_{t^{\prime}}, P_{t^{\prime \prime}} \notin \mathcal{K} \cup \mathcal{W}$ such that $P_{t^{\prime}}$ intersects some path $P_{u^{\prime}} \in \mathcal{K}$ at the left of $x_{1}$ and $P_{t^{\prime \prime}}$ intersects some path $P_{u^{\prime \prime}} \in \mathcal{K}$ at the right of $x_{1}$. Indeed, every path $P_{w} \in \mathcal{W}$ must intersect all these paths $P_{t}, P_{t^{\prime}}, P_{t^{\prime \prime}}$ which is clearly not possible since the paths are single-bended and $t, t^{\prime}, t^{\prime \prime} \notin K \cup W$. Thus we may assume that there is no path $P_{t^{\prime \prime}} \notin \cap K \cup W$ intersecting some path $P_{u^{\prime \prime}} \in \mathcal{K}$ at the right of $x_{1}$.

First assume that there exists a path $P_{t^{\prime}} \notin \mathcal{K} \cup \mathcal{W}$ intersecting some path $P_{u} \in \mathcal{K}$ at the left of $x_{1}$. Notice that $P_{t}$ and $P_{t^{\prime}}$ cannot intersect because $t, t^{\prime} \notin K \cup W$ and because all paths are single-bended. Furthermore no path $P_{u} \in \mathcal{K}$ intersects both $P_{t}$ and $P_{t^{\prime}}$, otherwise $\left(u ; t, t^{\prime}, v\right)$ is a claw, a contradiction. Also all paths $P_{w} \in \mathcal{W}$ must be $\lrcorner$-paths in order to intersect both $P_{t}$ and $P_{t^{\prime}}$. Now distinguish two cases: (i) if $P_{u^{\prime}} \in \mathcal{K}$ is a $\lrcorner$-path and intersects $P_{t}$, then we may assume that its leftmost endpoint is $\left(x_{0}, y_{0}\right)$; now transform $P_{u^{\prime}}$ into a $\left\llcorner\right.$-path with rightmost endpoint $\left(x_{2}, y_{0}\right)$; (ii) if $P_{u^{\prime}} \in \mathcal{K}$ is a $\lrcorner$-path and intersects $P_{t^{\prime}}$, then delete its vertical part and add the horizontal segment $\left[x_{1}, x_{2}\right] \times\left\{y_{0}\right\}$; but now all the paths $P \in \mathcal{K}$ use the horizontal segment $\left[x_{1}, x_{2}\right] \times\left\{y_{0}\right\}$ and no other path uses it; thus we may add $P_{v}$ on that segment to obtain a $B_{1}$-EPG representation of $G$, a contradiction.

Finally consider the case where there exists a path $P_{t} \notin \mathcal{K} \cup \mathcal{W}$ such that $P_{t}$ intersects a path $P_{u} \in \mathcal{K}$ on column $x_{1}$, but there exist no path $P_{t^{\prime}} \notin \mathcal{K} \cup \mathcal{W}$ intersecting a path $P_{u^{\prime}} \in \mathcal{K}$ at the left of $x_{1}$ or at the right of $x_{1}$. Clearly all paths $P_{w} \in \mathcal{W}$ are bended at $\left(x_{1}, y_{0}\right)$ in order to intersect $P_{t}$. If all the paths $P_{w} \in \mathcal{W}$ are $\lrcorner$-paths (resp. $\llcorner$-paths), we may proceed as in the previous case. Thus we may assume that there exists $P_{w} \in \mathcal{W}$ which is a $\lrcorner$-path and there exists $P_{w^{\prime}} \in \mathcal{W}$ which is a $\llcorner$-path. We may also assume that for every path $P_{u} \in \mathcal{K}$, either it is a $\left\llcorner\right.$-path and intersects $P_{t}$, or it is non-bended. This follows from our hypotheses and from the fact that if $P_{u} \in \mathcal{K}$ is a $\lrcorner$-path and intersects $P_{t}$, we may transform it into a $\llcorner$-path with rightmost endpoint $\left(x_{2}, y_{0}\right)$. But now we may delete the horizontal parts from all the bended paths $P_{u} \in \mathcal{K}$ (recall that no path $P_{u} \in \mathcal{K}$ intersects a path $P_{t^{\prime}} \notin \mathcal{K} \cup \mathcal{W}$ at the left of $x_{1}$ or the right of $x_{1}$ ) and we may transform all non-bended $P_{u}$ paths (for which we may assume that their endpoints are $\left(x_{0}, y_{0}\right)$ and $\left(x_{2}, y_{0}\right)$ ) into non-bended paths using the grid segment $\left\{x_{1}\right\} \times\left[y_{0}, y_{1}\right]$. Thus $K \cup W$ is represented as an edge clique and we are done.

This proves the case where $K \cup W$ is a claw clique. 


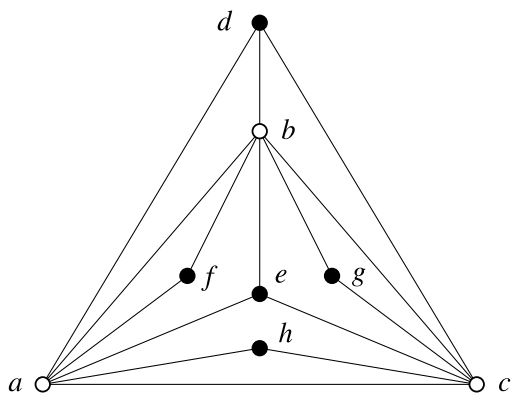

Fig. 11. The split graph $G_{0}$.

\subsection{Chordal diamond-free graphs}

A diamond is a graph with vertex set $\{a, b, c, d\}$ and edge set $\{a b=a c, b c, b d, c d\}$. A graph is diamond-free if it does not contain any induced subgraph isomorphic to the diamond.

We will first start with an easy observation which will be helpful in the proof of the main result of this subsection.

Lemma 18. Let $G$ be a $C_{4}$-free $B_{1}-E P G$ graph and let $v \in V(G)$ be a simplicial vertex. Then there exists a $B_{1}-E P G$ representation of $G$ in which $P_{v}$ is a non-bended path.

Proof. Consider a $B_{1}$-EPG representation of $G \backslash\{v\}$. If the clique $N(v)$ is represented as an edge clique, then it follows from Lemma 14(1) that we may assume that there exists an edge $e$ in the grid only used by paths corresponding to vertices of $N(v)$. Now $P_{v}$ may be added such that it coincides with exactly this edge $e$. If the clique $N(v)$ is represented as a claw clique, then it follows from Lemma 14(2) that we may assume that only paths corresponding to vertices of $N(v)$ use some row $y_{j}$ between columns $x_{i-1}$ and $x_{i+1}$, for some $i$ and $j$. Now $P_{v}$ may be added such that it uses row $y_{j}$ between columns $x_{i-1}$ and $x_{i+1}$.

Theorem 19. Every chordal diamond-free graph $G$ is a $B_{1}-E P G$ graph.

Proof. Suppose the result is false and let $G^{\prime}$ be a minimal counterexample. Let $v \in V\left(G^{\prime}\right)$ be a simplicial vertex. Denote by $K$ the clique induced by $N(v)$. As in the proof of Theorem 17, we are done if $K$ is maximal in $G^{\prime} \backslash\{v\}$. Thus we may assume that $K$ is not maximal, i.e., there exists at least one vertex $w \in V\left(G^{\prime}\right) \backslash(K \cup\{v\})$ which is complete to $K$. This implies that $|N(v)|=1$. Indeed, if $u_{1}, u_{2} \in N(v)$, we get a diamond induced by $\left\{v, w, u_{1}, u_{2}\right\}$, a contradiction. So let $N(v)=\{u\}$ and consider a $B_{1}$-EPG representation of $G^{\prime \prime}=G^{\prime} \backslash\{v\}$. Clearly the graph $G_{u}=G^{\prime \prime} \mid N(u)$ must be connected, otherwise there exists a grid point $\left(x_{i}, y_{j}\right)$ such that $P_{u}$ is the only path using that grid point. But then we may assume that there is an edge either on column $x_{i}$ or on row $y_{j}$ which is only used by $P_{u}$ (we may have to shift some paths to obtain this configuration). Thus we may add $P_{v}$ on that particular edge and get a $B_{1}$-EPG representation of $G^{\prime}$, a contradiction. So we may assume that $G_{u}$ is connected. Furthermore, $G_{u}$ must be a clique. Indeed, if $z, z^{\prime} \in V\left(G_{u}\right)$ and $z z^{\prime} \notin E\left(G_{u}\right)$, then there must be an induced path $\left\{z z_{1}, z_{1} z_{2}, \ldots, z_{k} z^{\prime}\right\}$ in $G_{u}$ from $z$ to $z^{\prime}$ since $G_{u}$ is connected. But now $\left\{u, z, z_{1}, z_{2}\right\}$ induce a diamond, a contradiction. Thus $u$ is a simplicial vertex in $G^{\prime \prime}$. It follows from Lemma 18 that we may assume that $P_{u}$ is represented as a non-bended path in the $B_{1}$-EPG representation of $G^{\prime \prime}$. Suppose $P_{u}$ uses some row $y_{k}$ between columns $x_{i}$ and $x_{j}, i<j$. Next we insert an additional column $x_{i^{\prime}}$ between $x_{i}$ and $x_{i+1}$. Clearly no path uses column $x_{i^{\prime}}$. Now we delete the horizontal part of $P_{u}$ between columns $x_{i}$ and $x_{i^{\prime}}$ and we add a vertical part on column $x_{i^{\prime}}$ between rows $y_{k}$ and $y_{k-1}\left(P_{u}\right.$ becomes a $\left\ulcorner\right.$-path with bend point $\left.\left(x_{i^{\prime}}, y_{k}\right)\right)$. Hence we may represent $P_{v}$ on that vertical part. Thus we obtain a $B_{1}$-EPG representation of $G^{\prime}$, which is a contradiction.

\subsection{Split graphs}

A split graph is a graph $G$ such that $V(G)$ can be partitioned into a clique $K$ and a stable set $S$. In [11] the following characterization of split graphs is given. A graph $G$ is a split graph if and only if $G$ is $\left\{H_{4}, H_{5}, \bar{H}_{4}\right\}$-free. It is easy to see that all split graphs with either $|K| \leq 2$ or $|S| \leq 2$ are $B_{1}$-EPG. Here we will consider split graphs for which either $|K|=3$ or $|S|=3$ and we will give a characterization of those that are $B_{1}$-EPG. We may assume, without loss of generality, that the split graphs $G$ that we consider are connected.

In the following figures, the vertices of the clique $K$ will be represented as white nodes (in some figures we represent a clique by a shaded area instead of drawing the edges of the clique) and the vertices of the independent set $S$ will be represented as black nodes. First we consider the split graph $G_{0}$ represented in Fig. 11. We obtain the following result.

Lemma 20. The graph $G_{0}$ is not $B_{1}-E P G$.

Proof. Suppose for a contradiction that $G_{0}$ is $B_{1}$-EPG and consider a $B_{1}$-EPG representation. First assume that the clique $K$ is represented as a claw clique with center $\left(x_{i}, y_{j}\right)$. It follows that $P_{d}$ and $P_{e}$ must both contain the grid point $\left(x_{i}, y_{j}\right)$ and intersect all three paths $P_{a}, P_{b}$ and $P_{c}$. But then $P_{d}$ and $P_{e}$ necessarily intersect, which is a contradiction. 

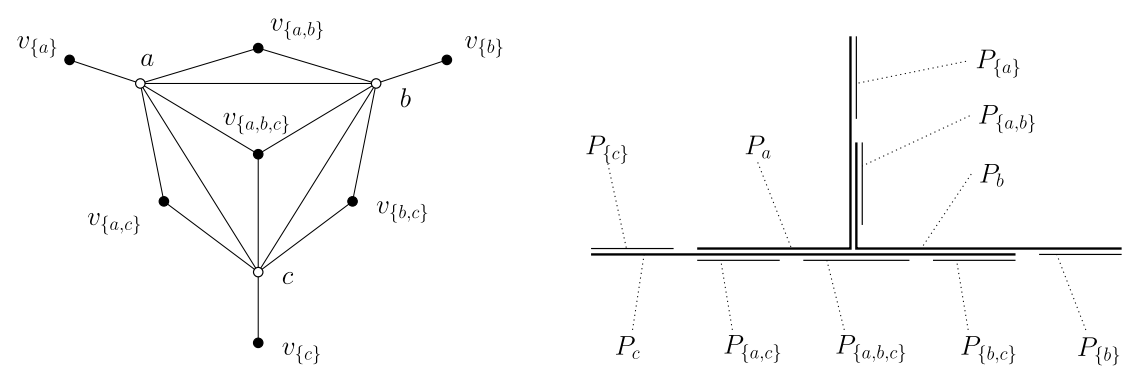

Fig. 12. Split graph $G$ with $|K|=3$ (a path corresponding to $v_{J}$ is denoted by $P_{J}$, rather than by $P_{v_{J}}$ ).
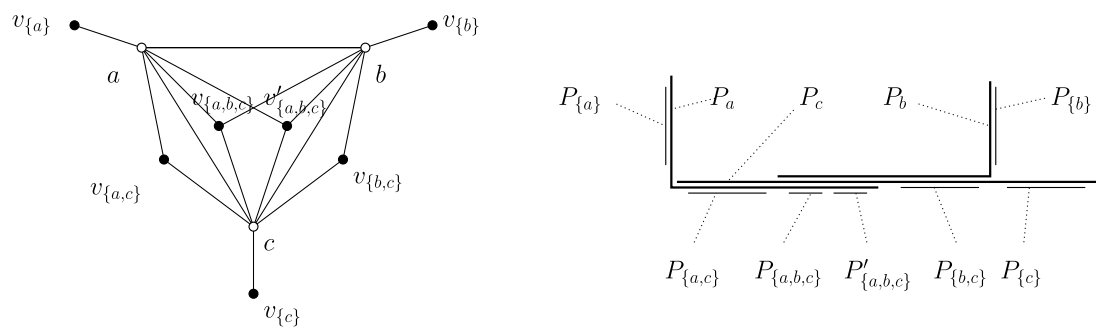

Fig. 13. Split graph $G$ with $|K|=3$; case where $\left|N_{\{a, b, c\}}\right| \geq 2$ and $N_{\{a, b\}}=\emptyset$.

Thus we may assume now that $K$ is represented as an edge clique. Let $\left[x_{i}, x_{i+1}\right] \times\left\{y_{j}\right\}$ be an edge on the grid contained in all three paths $P_{a}, P_{b}, P_{c}$. Notice that $G_{0} \mid\{a, b, c, f, g, h\}$ is isomorphic to the 3-sun $S_{3}$ which is not an interval graph since $f, g, h$ form an asteroidal triple (see [15]). Thus at least one path of $P_{a}, P_{b}, P_{c}$ must be bended and intersect a path of $P_{f}, P_{g}, P_{h}$ on a column $x_{k}$. Without loss of generality, we may assume that $P_{f}$ uses column $x_{k}$ and intersects $P_{a}$ on that column, with $k<i$.

Let us now distinguish two cases. First assume that $P_{b}$ also intersects $P_{f}$ on column $x_{k}$. Then necessarily $P_{g}$ must intersect $P_{b}$ and $P_{c}$ on row $y_{j}$ at the right of $x_{i+1}$. But now clearly $P_{h}$ cannot intersect $P_{a}$ and $P_{c}$ without intersecting any other path. Thus we may assume now that $P_{b}$ intersects $P_{f}$ on row $y_{j}$. Hence $G_{0} \mid\{a, b, f\}$ is represented as a claw clique with center $\left(x_{k}, y_{j}\right)$. It follows that both $P_{g}$ and $P_{h}$ must lie at the right of $x_{i+1}$ and in fact $P_{h}$ must intersect $P_{a}$ and $P_{c}$ on row $y_{j}$. But now $P_{g}$ cannot intersect $P_{b}$ and $P_{c}$ without intersecting any other path. Thus we conclude that $G_{0}$ is not $B_{1}$-EPG.

We are now ready to give a characterization of all split graphs with $|K|=3$ that are $B_{1}$-EPG.

Theorem 21. Let $G$ be a split graph with $V(G)=K \cup S, K \cap S=\emptyset$, where $K$ is a clique, $S$ is a stable set and $|K|=3$. Then $G$ is $B_{1}-E P G$ if and only if $G$ is $G_{0}$-free.

Proof. It follows from Lemma 20 that $G_{0}$ is not $B_{1}$-EPG. Let $G$ be a split graph with $|K|=3$ and not containing any induced subgraph isomorphic to $G_{0}$. Let $a, b, c$ be the vertices of $K$. For each $J \subseteq\{a, b, c\}$, denote by $N_{J}$ the set of vertices of $S$ that are adjacent only to the members of $J$. We may assume that $N_{\emptyset}=\emptyset$. Indeed it is always possible to add isolated paths to a $B_{1}$-EPG representation. Assume first that for each nonempty $J \subseteq\{a, b, c\}$, we have $\left|N_{J}\right|=1$; define $N_{J}=\left\{v_{J}\right\}$. Then we obtain the graph represented in Fig. 12 and a feasible $B_{1}$-EPG representation of it.

Consider now the general case, i.e., $\left|N_{J}\right| \geq 0$ for all nonempty sets $J \subseteq\{a, b, c\}$. We will distinguish two subcases.

If $\left|N_{\{a, b, c\}}\right| \leq 1$, we modify the previous construction as follows. For every $\emptyset \neq J \subseteq\{a, b, c\}$ such that $N_{J}=\emptyset$, we just delete $P_{J}$; for every $\emptyset \neq J \subset\{a, b, c\}$ such that $\left|N_{J}\right|>1$, we split the path $P_{J}$ into $\left|N_{J}\right|$ non-overlapping segments.

If $\left|N_{\{a, b, c\}}\right| \geq 2$, we proceed as follows. Since $G$ is $G_{0}$-free, at least one of the sets $N_{\{a, b\}}, N_{\{b, c\}}, N_{\{a, c\}}$ is empty. Without loss of generality, we may assume that $N_{\{a, b\}}=\emptyset$. Then we clearly obtain a feasible $B_{1}$-EPG representation of $G$ as shown in Fig. 13. Notice that, for the sake of simplicity, we took $\left|N_{\{a, b, c\}}\right|=2$ and $\left|N_{J}\right|=1$ for each $J \subseteq\{a, b, c\}, J \neq\{a, b, c\},\{a, b\}$; we can easily transform this representation if we have $\left|N_{J}\right|>1$ for some sets $J \subseteq\{a, b, c\}, J \neq\{a, b, c\},\{a, b\}$, by splitting the corresponding paths $P_{J}$ into $\left|N_{J}\right|$ non-overlapping segments (as mentioned already above).

Let us now consider split graphs with $|S|=3$. Consider the three split graphs $G_{1}, G_{2}, G_{3}$ shown in Fig. 14 . Notice that in all these graphs the vertices of $S$ form an asteroidal triple contained in the neighborhood of the vertex denoted by $v$. It follows from Theorem 9 that $G_{1}, G_{2}$ and $G_{3}$ are not $B_{1}$-EPG. In particular, these graphs show that in Theorem $21|K|=3$ cannot simply be replaced by $|K|=4$.

We obtain the following result.

Theorem 22. Let $G$ be a split graph with $V(G)=K \cup S, K \cap S=\emptyset$, where $K$ is a clique, $S$ is a stable set and $|S|=3$. Then $G$ is $B_{1}-E P G$ if and only if $G$ is $\left\{G_{1}, G_{2}, G_{3}\right\}$-free. 


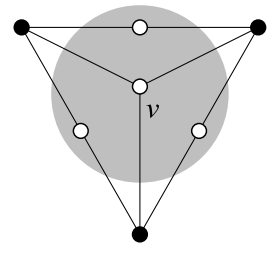

$G_{1}$

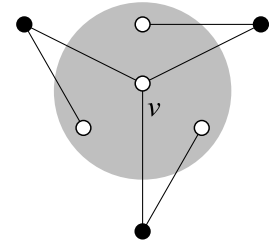

$G_{2}$

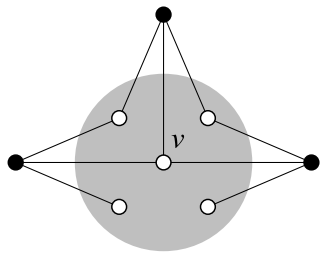

$G_{3}$

Fig. 14. The graphs $G_{1}, G_{2}, G_{3}$.
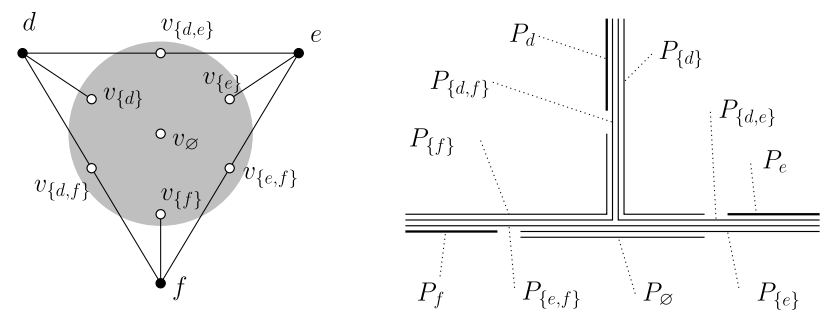

Fig. 15. Split graph $G$ with $|S|=3, N_{\{d, e, f\}}=\emptyset$.
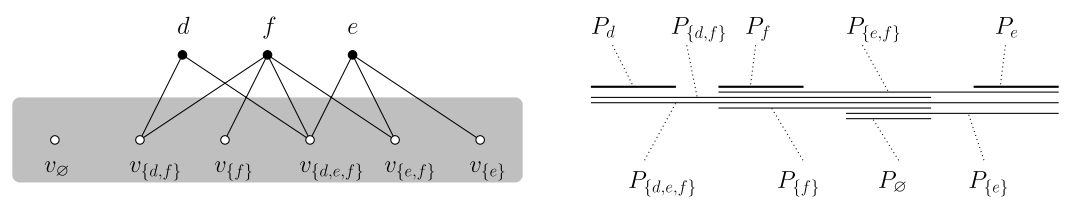

Fig. 16. Split graph $G$ with $|S|=3, N_{\{d, e, f\}} \neq \emptyset, N_{\{d, e\}}=N_{\{d\}}=\emptyset$.

(2a)

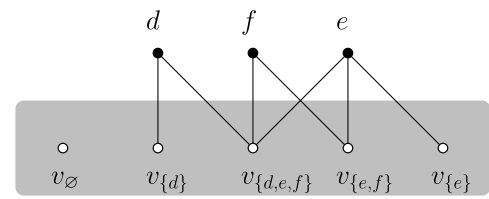

(2b)

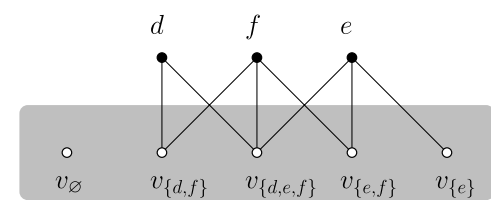

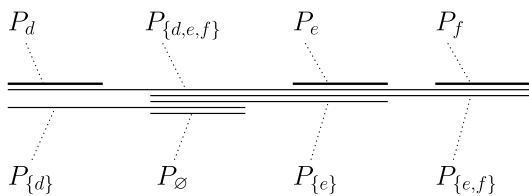

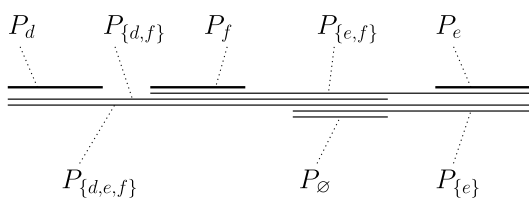

Fig. 17. Split graph $G$ with $|S|=3, N_{\{d, e, f\}} \neq \emptyset, N_{\{d, e\}}=N_{\{f\}}=\emptyset$.

Proof. Let $d, e, f$ be the vertices of $S$. For each $J \subseteq\{d, e, f\}$, denote by $N_{J}$ the set of vertices of $K$ that are adjacent only to the members of $J$. Assume first that $N_{\{d, e, f\}}=\emptyset$. We may assume that for each $J \varsubsetneqq\{d, e, f\}$ we have $\left|N_{J}\right|=1$. Let $N_{J}=\left\{v_{J}\right\}$ (otherwise we shall duplicate or delete the corresponding paths in the following construction). Then we obtain the graph represented in Fig. 15 and a $B_{1}$-EPG representation of it.

Assume now that $N_{\{d, e, f\}} \neq \varnothing$. At least one among the sets $N_{\{d, e\}}, N_{\{d, f\}}, N_{\{e, f\}}$ is empty since otherwise $G$ contains an induced subgraph isomorphic to $G_{1}$. Moreover, at least one among the sets $N_{\{d\}}, N_{\{e\}}, N_{\{f\}}$ is empty since otherwise $G$ contains an induced subgraph isomorphic to $G_{2}$. Thus there are, up to relabeling, two cases to distinguish: (1) $N_{\{d, e\}}=N_{\{d\}}=\emptyset$; (2) $N_{\{d, e\}}=N_{\{f\}}=\emptyset$.

In the first case, we may assume that for all $J \subseteq\{d, e, f\}, J \neq\{d, e\},\{d\}$, we have $\left|N_{J}\right|=1, N_{J}=\left\{v_{J}\right\}$. Then $G$ has a $B_{1}$-EPG representation as shown in Fig. 16.

In the second case, it is impossible that all the sets $N_{\{d, e, f\}}, N_{\{d, f\}}, N_{\{e, f\}}, N_{\{d\}}, N_{\{e\}}$ are nonempty since otherwise $G$ contains an induced subgraph isomorphic to $G_{3}$. Then we have essentially two cases: (2a) $N_{\{d, f\}}=\emptyset$ and (2b) $N_{\{d\}}=\emptyset$ (recall that we are dealing with the case $N_{\{d, e, f\}} \neq \emptyset$ ). In both cases we assume that for all the sets $N_{J}$ (except those assumed to be empty) we have $\left|N_{J}\right|=1, N_{J}=\left\{v_{J}\right\}$ and obtain a $B_{1}$-EPG representation of $G$ as shown in Fig. 17. 
Notice that in the case where $N_{\{d, e, f\}} \neq \emptyset$, we could (instead of giving a $B_{1}$-EPG representation) note that $G$ is a chordal AT-free graph and therefore it is an interval graph, and hence $B_{1}$-EPG.

\section{Conclusion}

In this paper we considered edge intersection graphs of single-bend paths on a grid ( $B_{1}$-EPG graphs). We showed that in $B_{1}$-EPG graphs the subgraph induced by the neighborhood of any vertex is weakly chordal, and thus perfect. This allowed us to prove that these graphs satisfy the Erdős-Hajnal property, i.e., they contain either a large clique or a large stable set. Then we considered some subclasses of chordal graphs and characterized the $B_{1}$-EPG graphs of these subclasses. Some of these results are constructive and explain how to obtain a $B_{1}$-EPG representation if one exists.

There remain a lot of open questions concerning $B_{1}$-EPG graphs and more generally $B_{k}$-EPG graphs for $k \geq 1$. For instance it would be interesting if one could characterize $B_{1}$-EPG graphs of special classes of graphs other than those presented here. Chordal graphs would be of major interest. Furthermore, a question arising from our results is that of whether $\frac{1}{3}$ is best possible for the Erdős-Hajnal property for $B_{1}$-EPG graphs.

Finally let us mention that it is still unknown whether, for instance, the vertex coloring problem or the maximum clique problem is polynomially solvable in $B_{1}$-EPG graphs.

\section{Acknowledgments}

The present work was partially done while the second author was a postdoctoral research fellow at Columbia University, an assistant professor at the University of Warwick (DIMAP Center) and a visiting research fellow at the University of Haifa (Caesarea Rothschild Institute). The support of these institutions is gratefully acknowledged.

Furthermore, the authors would like to thank two anonymous referees for their valuable comments and remarks that helped with improving the paper.

\section{References}

[1] N. Alon, J. Pach, R. Pinchasi, R. Radoičić, M. Sharir, Crossing patterns of semi-algebraic sets, Journal of Combinatorial Theory, Series A 111 (2005) 310-326.

[2] A. Asinowski, A. Suk, Edge intersection graphs of systems of paths on a grid with a bounded number of bends, Discrete Applied Mathematics 157 (2009) 3174-3180.

[3] M.L. Bandy, M. Sarrafzadeh, Stretching a knock-knee layout for multilayer wiring, IEEE Transactions on Computers 39 (1990) 148-151

[4] C. Berge, Graphs and Hypergraphs, North-Holland Publishing Company, Amsterdam, 1973.

[5] C. Berge, Problèmes de coloration en théorie des graphes, Publications de l'Institut de Statistique de l'Université de Paris 9 (1960) 123-160.

[6] T. Biedl, M. Stern, On edge intersection graphs of $k$-bend paths in grids, Discrete Mathematics and Theoretical Computer Science 12 (1) (2010) 1-12.

[7] M. Chudnovsky, N. Robertson, P. Seymour, R. Thomas, The strong perfect graph theorem, Annals of Mathematics 164 (2006) 51-229.

[8] D.G. Corneil, S. Olariu, L. Stewart, Asteroidal triple-free graphs, Technical Report, University of Toronto, 1992.

[9] G.A. Dirac, On rigid circuit graphs, Abhandlungen aus dem Mathematischen Seminar der Universität Hamburg 25 (1961) 71-76.

[10] P. Erdős, A. Hajnal, Ramsey-type theorems, Discrete Applied Mathematics 25 (1989) 37-52.

[11] S. Földes, P.S. Hammer, Split graphs, in: F. Hoffman et al. (Eds.) Proceedings of the 8th Southeastern Conference on Combinatorics, Graph Theory and Computing, 1977, pp. 311-315.

[12] J. Fox, J. Pach, C.D. Tóth, Intersection patterns of curves, Journal of the London Mathematical Society 83 (2011) 389-406

[13] M.C. Golumbic, M. Lipshteyn, M. Stern, Edge intersection graphs of single bend paths on a grid, Networks 54 (3) (2009) $130-138$.

[14] D. Heldt, K. Knauer, T. Ueckerdt, Edge-intersection graphs of grid paths: the bend number, http://arxiv.org/abs/1009.2861v1.

[15] C. Lekkerkerker, D. Boland, Representation of finite graphs by a set of intervals on the real line, Fundamenta Mathematicae 51 (1969) 45-64.

[16] B. Reed, N. Sbihi, Recognizing bull-free graphs, Graphs and Combinatorics 11 (1995) 171-178. 